\title{
Generalization of conditioned fear along a dimension of increasing fear intensity
}

\author{
Joseph E. Dunsmoor, Stephen R. Mitroff, and Kevin S. LaBar ${ }^{1}$ \\ Center for Cognitive Neuroscience and Department of Psychology and Neuroscience, Duke University, Durham, North Carolina \\ 27708, USA
}

\begin{abstract}
The present study investigated the extent to which fear generalization in humans is determined by the amount of fear intensity in nonconditioned stimuli relative to a perceptually similar conditioned stimulus. Stimuli consisted of graded emotionally expressive faces of the same identity morphed between neutral and fearful endpoints. Two experimental groups underwent discriminative fear conditioning between a face stimulus of $55 \%$ fear intensity (conditioned stimulus, $\mathrm{CS}+$ ), reinforced with an electric shock, and a second stimulus that was unreinforced (CS-). In Experiment 1 the $C S-$ was a relatively neutral face stimulus, while in Experiment 2 the CS- was the most fear-intense stimulus. Before and following fear conditioning, skin conductance responses (SCR) were recorded to different morph values along the neutral-to-fear dimension. Both experimental groups showed gradients of generalization following fear conditioning that increased with the fear intensity of the stimulus. In Experiment 1 a peak shift in SCRs extended to the most fear-intense stimulus. In contrast, generalization to the most fear-intense stimulus was reduced in Experiment 2, suggesting that discriminative fear learning procedures can attenuate fear generalization. Together, the findings indicate that fear generalization is broadly tuned and sensitive to the amount of fear intensity in nonconditioned stimuli, but that fear generalization can come under stimulus control. These results reveal a novel form of fear generalization in humans that is not merely based on physical similarity to a conditioned exemplar, and may have implications for understanding generalization processes in anxiety disorders characterized by heightened sensitivity to nonthreatening stimuli.
\end{abstract}

Fear generalization occurs when a fear response acquired to a particular stimulus transfers to another stimulus. Generalization is often an adaptive function that allows an organism to rapidly respond to novel stimuli that are related in some way to a previously learned stimulus. Fear generalization, however, can be maladaptive when nonthreatening stimuli are inappropriately treated as harmful, based on similarity to a known threat. For example, an individual may acquire fear of all dogs after an aversive experience with a single vicious dog. In this case, recognizing that a novel animal is related to a feared (or fearconditioned) animal is made possible in part by shared physical features to the fear exemplar, such as four legs and a tail. On the other hand, fear generalization may be selective for those features that are associated with natural categories of threat; a harmless dog may not pose a threat, but possesses naturally threatening features common to other threatening animals, such as sharp teeth and claws. Moreover, the degree to which an individual fearful of dogs responds with fear may be related to either the physical similarity to the originally feared animal (e.g., from a threatening black dog to another black dog), or the intensity of those threatening features relative to the originally feared animal (e.g., sharp teeth from one animal to sharp teeth of another animal). Therefore, fear generalization based on perceptual information may occur via two routes-similarity to a learned fear exemplar along nonthreatening physical dimensions or along dimensions of fear relevance. Given that fear generalization often emerges as a consequence of conditioning or observational learning, it is important to determine which characteristics of novel stimuli facilitate fear generalization and the extent to which generalization processes can be controlled.

\footnotetext{
'Corresponding author.
}

E-mail klabar@duke.edu; fax (919) 681-0815.

Article is online at http://www.learnmem.org/cgi/doi/10.1101/lm.1431609.
Early explanations of stimulus generalization emphasized that an organism's ability to generalize to nonconditioned stimuli is related to both the similarity and discriminability to a previously conditioned stimulus (CS) (Hull 1943; Lashley and Wade 1946). While Lashley and Wade (1946) argued that generalization was simply a failure of discriminating between a nonconditioned stimulus (CS-) and the reinforced CS (CS+), contemporary views contend that generalization enables learning to extend to stimuli that are readily perceptually distinguished from the CS (Pearce 1987; Shepard 1987; McLaren and Mackintosh 2002). This latter view has been supported by empirical studies of stimulus generalization in laboratory animals (Guttman and Kalish 1956; Honig and Urcuioli 1981). In these studies, animals were reinforced for responding to a CS of a specific physical quality such as color, and then tested with several different values along the same stimulus dimension as the CS (e.g., at various wavelengths along the color spectrum). Orderly gradients of responses are often reported that peak at or near the reinforced value and decrease as a function of physical similarity to the CS along the stimulus dimension (Honig and Urcuioli 1981). Further generalization was shown to extend from the CS+ to discriminable nonconditioned stimuli, suggesting that generalization is not bound to the organism's ability to discriminate stimuli (Guttman and Kalish 1956, 1958; Shepard 1987).

Interestingly, when animals learn to distinguish between a CS+ and a CS -, the peak of behavioral responses often shift to a new value along the dimension that is further away from the CS- (Hanson 1959). For instance, when being trained to discriminate a green $\mathrm{CS}+$ and an orange $\mathrm{CS}_{-}$, pigeons will key peck more to a greenish-blue color than the actual CS+ hue. Intradimensional generalization of this sort is reduced when animals are trained to discriminate between two or more stimulus values that are relatively close during conditioning (e.g., discriminating a greenyellow CS+ from a green-blue CS-), suggesting that the extent of generalization can come under stimulus control through 
reinforcement learning (Jenkins and Harrison 1962). Spence (1937) described the transposition of response magnitude as an effect of interacting gradients of excitation and inhibition formed around the CS+ and CS-, respectively, which summate to shift responses to values further from the inhibitory CS - gradient. In all, early theoretical and empirical treatments of stimulus generalization in nonhuman animals revealed that behavior transfers to stimuli that are physically similar, but can be discriminated from a CS, and that differential reinforcement training can both sharpen the stimulus gradient and shift the peak of responses to a nonreinforced value.

Although this rich literature has revealed principles of generalization in nonhuman animals, few studies of fear generalization have been conducted in humans (for review, see Honig and Urcuioli 1981; Ghirlanda and Enquist 2003). Moreover, the existing human studies have yet to consider the second route through which fear responses may generalize-via gradients of fear relevance. While a wide range of neutral stimuli, such as tones or geometric figures, can acquire fear relevance through conditioning processes, other stimuli, such as threatening faces or spiders, are biologically prepared to be fear relevant (Lanzetta and Orr 1980; Dimberg and Öhman 1996; Whalen et al. 1998; Öhman and Mineka 2001). Compared with fear-irrelevant CSs, biologically prepared stimuli capture attention (Öhman et al. 2001), are conditioned without awareness (Öhman et al. 1995; Öhman and Soares 1998), increase brain activity in visual and emotional processing regions (Sabatinelli et al. 2005), and become more resistant to extinction when paired with an aversive unconditioned stimulus (US) (Öhman et al. 1975). Although the qualitative nature of the CS influences acquisition and expression of conditioned fear, it is unknown how generalization proceeds along a gradient of natural threat. For instance, human studies to date have all tested variations of a CS along physically neutral stimulus dimensions, such as tone frequency (Hovland 1937), geometric shape (Vervliet et al. 2006), and physical size (Lissek et al. 2008). These investigations implicitly assume that the generalization gradient is independent of the conditioned value (equipotentiality principle). In other words, since the stimuli are all equally neutral prior to fear learning, fear generalization operates solely as a function of similarity along the reinforced physical dimension. However, since fear learning is predisposed toward fear-relevant stimuli, generalization may be selective to those shared features between a CS+ and CS- that are associated with natural categories of threat. Examining generalization using fearrelevant stimuli is thus important to gain better ecological validity and to develop a model system for studying maladaptive fear generalization in individuals who may express exaggerated fear responses to nonthreatening stimuli following a highly charged aversive experience (i.e., post-traumatic stress disorder or specific phobias).

To address this issue, the present study examined generalization to fearful faces along an intradimensional gradient of fear intensity. A fearful face is considered a biologically prepared stimulus that recruits sensory systems automatically for rapid motor responses (Öhman and Mineka 2001), and detecting fearful faces may be evolutionarily selected as an adaptive response to social signals of impending danger (Lanzetta and Orr 1980; Dimberg and Öhman 1996). During conditioning, an ambiguous face containing $55 \%$ fear intensity (CS+) was paired with an electric shock US, while a relatively neutral face (11\% fear intensity) was explicitly unreinforced (CS-) (Experiment 1). Skin conductance responses (SCR) were recorded as a dependent measure of fear conditioning. Before and following fear conditioning, SCRs were recorded in response to face morphs of the same actor expressing several values of increasing fear intensity (from $11 \%$ to 100\%; see Fig. 1). A total of five values along the continuum were used: $11 \%$ fear $/ 88 \%$ neutral, 33\% fear/66\% neutral, 55\% fear $/ 44 \%$ neutral, $77 \%$ fear $22 \%$ neutral, and $100 \%$ fear. For clarity, these stimuli are herein after labeled as S1, S2, S3, S4, and S5, respectively.

Testing generalization along an intradimensional gradient of emotional expression intensity allows for an examination of the relative contributions of fear intensity and physical similarity on the magnitude of generalized fear responses. If fear generalization is determined purely by the perceptual overlap between the CS+ and other morph values, without regard to fear intensity, then we would expect a bell-shaped generalization function with the maximum SCR centered on the reinforced (intermediate) CS+ value (S3), less responding to the directly adjacent, but most perceptually similar values (S2 and S4), and the least amount of responding to the most distal and least perceptually similar morph values (S1 and S5). This finding would be in line with stimulus generalization reported along fear-irrelevant dimensions (Lissek et al. 2008) and in stimulus generalization studies using appetitive instrumental learning procedures (Guttman and Kalish 1956). If, however, fear generalization is biased toward nonconditioned stimuli of high fear intensity, then an asymmetric generalization function should result with maximal responding to the most fearintense nonconditioned stimuli. This finding would suggest that fear generalization is selective to the degree of fear intensity in stimuli, similar to studies of physical intensity generalization gradients in nonhuman animals (Ghirlanda and Enquist 2003). We predicted that the latter effect would be observed, such that
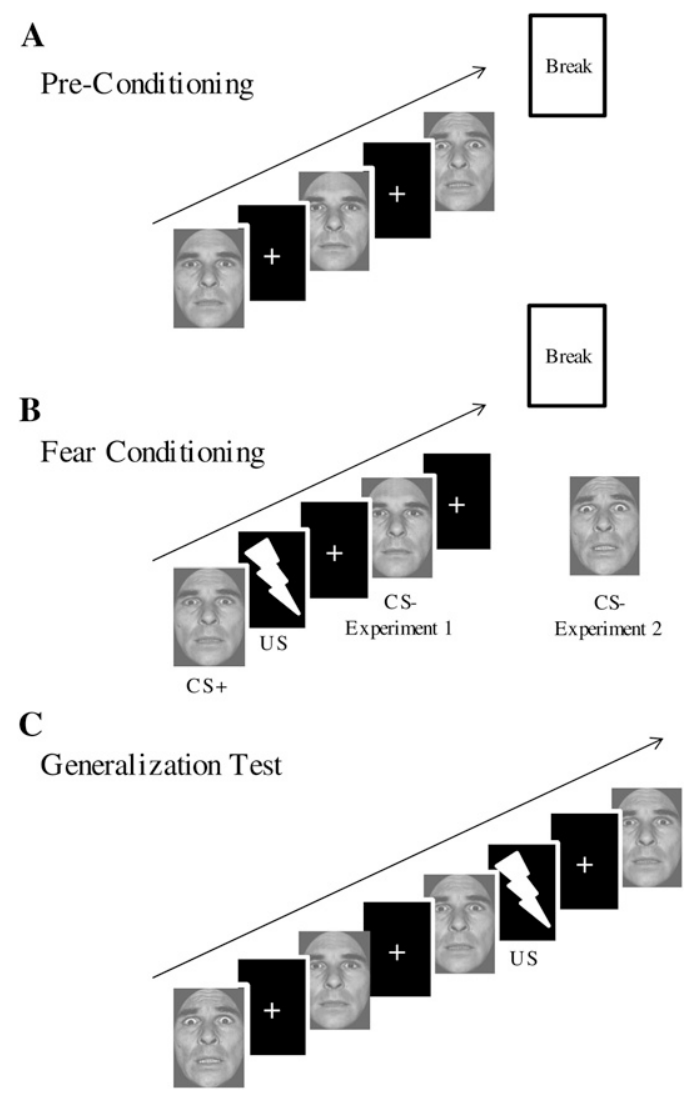

Figure 1. Experimental design. (A) Pre-conditioning included six presentations of all five stimulus values without the US. (B) Fear conditioning involved discriminative fear learning between the S3, paired with the US (CS+), and either the unreinforced S1 (Experiment 1) or the unreinforced S5 (Experiment 2) (CS-). (C) The generalization test included nine presentations of all five stimuli (45 total), with three out of nine S3 trials reinforced with the US. Stimuli are not drawn to scale. 
the magnitude of SCRs will disproportionately generalize to stimuli possessing a greater degree of fear intensity than the CS+ (Experiment 1). A secondary goal was to determine whether fear generalization to nonconditioned stimuli can be reduced through discriminative fear learning processes. Therefore, a second group of participants was run for whom the CS- was the $100 \%$ fearful face (Experiment 2). In this case, we predicted that discriminative fear conditioning between the CS+ (55\% intensity) and the most fear-intense nonconditioned stimulus would sharpen the generalization gradient around the reinforced CS+ value, and that responses to the most fear-intense stimulus would decrease relative to Experiment 1. Moreover, this discriminative fearlearning process may provide evidence that fear generalization is influenced by associative learning processes and is not exclusively driven by selective sensitization to stimuli of high fear relevance (Lovibond et al. 1993). Finally, we were interested to discover whether generalization processes would yield subsequent false memory for the intensity of the CS+ in a post-experimental retrospective report. In sum, the present study has implications for understanding how fear generalization is related to the degree of fear intensity of a nonconditioned stimulus, the extent to which discrimination training efforts can thwart the generalization process, and how fear generalization affects stimulus recognition.

\section{Results}

\section{Experiment 1}

\section{Preconditioning}

An ANOVA using the five fear intensity values as repeated measures demonstrated no main effect of the amount of fear intensity on SCR magnitude, $F_{(4,140)}=$ $0.374, P=0.82$. This result indicates undifferentiated responses to faces containing different levels of fear intensity prior to conditioning (Fig. 2A). A main effect of stimulus type on reaction time (RT) was revealed (Fig. 2C), $F_{(4,136)}=19.63, P<$ 0.001 , as well as both linear $(P<0.01)$ and quadratic trends $(P<0.01)$. The fastest RTs were elicited by the most intense fear face $(100 \%$, S5). The slowest RTs were observed for the ambiguous stimuli, S2 and S3. Finally, participants consistently rated the $\mathrm{S} 3, \mathrm{~S} 4$, and $\mathrm{S} 5$ as fear expressive, while the $\mathrm{S} 1$ and $\mathrm{S} 2$ were consistently not rated as fear expressive (Fig. 2D; see also Graham et al. 2007; Lim and Pessoa 2008). Based on these results, a categorical boundary was determined to exist between the S2 and S3 (point of subjective equality), such that faces of $55 \%$ fear intensity and greater were reliably discriminated from faces of less fear intensity that appeared neutral. Despite the fact that the S1 and S2 were categorically separate from the S3-S5, there were significant differences in RT between the S1 and S2 $(P<0.01)$, which suggests that these two within-category stimuli were perceptually discriminable. Similarly, there were significant differences in RTs for the S3, S4, and S5 as well $(P \mathrm{~s}<0.01)$.

\section{Fear conditioning}

As depicted in Figure 3, SCRs were significantly greater to the CS+ (mean \pm SEM: $0.72 \pm 0.05)$ than to the CS $-(0.38 \pm 0.04)$, indicating that conditioning took place, $t_{(35)}=7.78, P<0.01$. RTs were significantly slower to the CS+ $(989.73 \mathrm{~ms} \pm 43.06)$ relative to the CS $-(919.13 \mathrm{~ms} \pm 38.45), t_{(35)}=2.38, P=0.02$. Finally, participants rated the CS+ as expressing fear on $93 \%$ of trials, whereas the CS - was rated as expressing fear on $<1 \%$ of trials.

\section{Generalization test}

\section{SCR analysis}

Fear generalization was characterized by a linear trend, $F_{(1,35)}=$ $24.47, P<0.01$, while other polynomial trends were not significant $(P>0.22)$. Importantly, the amount of generalization to each stimulus was a function of fear intensity, and peaked at the most intense fear value, S5 (Fig. 2A). Repeated-measures ANOVA confirmed a significant main effect for fear intensity value on SCR magnitude, $F_{(4,140)}=10.37, P<0.01$. Post-hoc Bonferronicorrected $t$-tests were performed and revealed significant differences between S1 and S3 $(P<0.005)$ and between S2 and S3 $(P<$ $0.05)$, indicating a decrease in SCRs to stimuli of less fear intensity
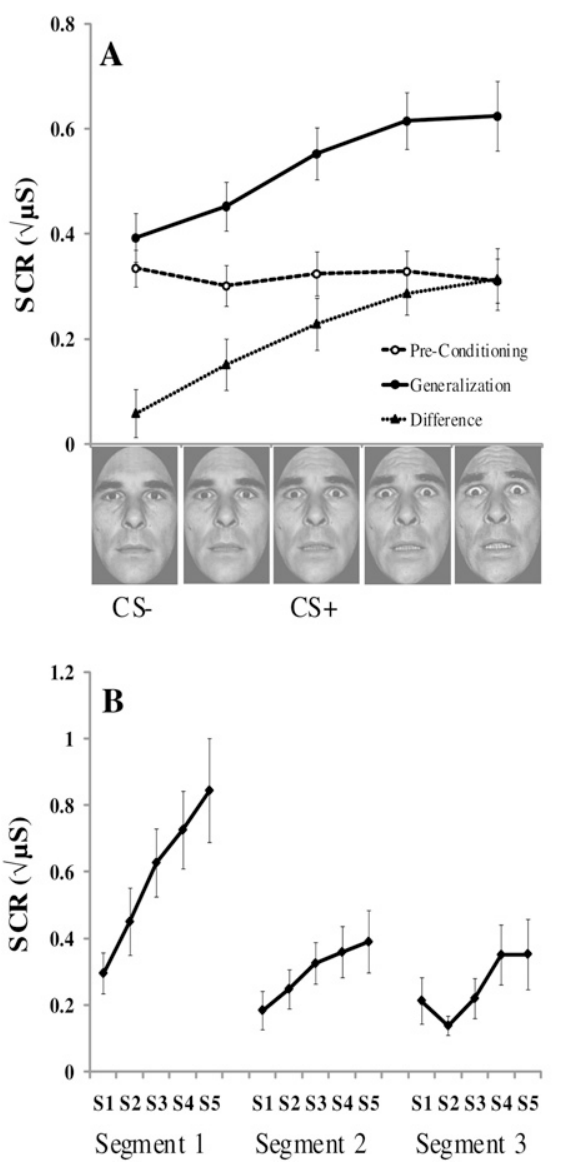
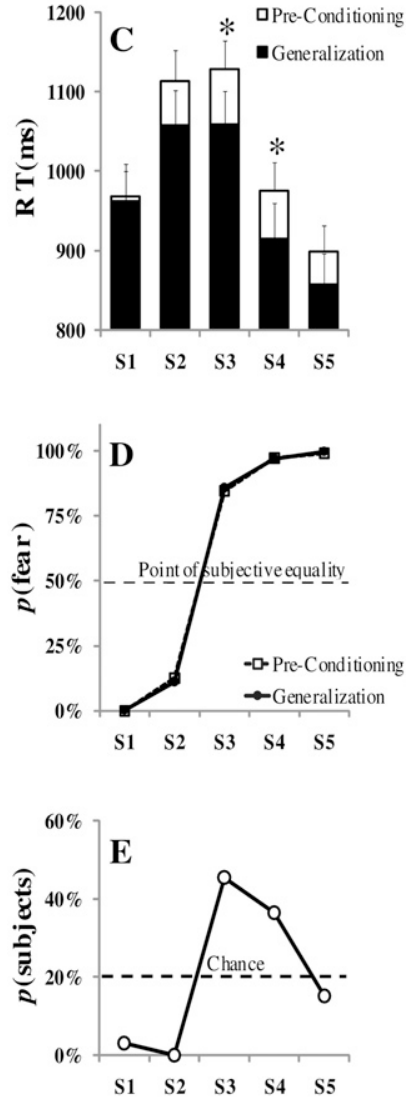

Figure 2. Experiment 1 results for preconditioning and the generalization test. $(A)$ Mean skin conductance responses (SCR) are undifferentiated to the stimuli before fear conditioning, but an asymmetrical linear generalization gradient emerges during the generalization test. $(B)$ Generalization across the three blocks of the generalization test shows that the pattern of generalized skin conductance responses was similar but habituated over time. (C) Reaction times (RT) were significantly faster following fear conditioning for the S3 and S4. (D) Subjective face ratings reveal a categorical boundary between the S2 and S3. (E) Retrospective CS+ identification demonstrates that a high percentage of subjects mistakenly identified the more fear-intense S4 as the CS+ post-experimentally. Error bars reflect standard error of the mean (SEM); $\left(^{*}\right)$ denote significant differences $(P<0.05) ;(\mu S)$ microsiemens. 


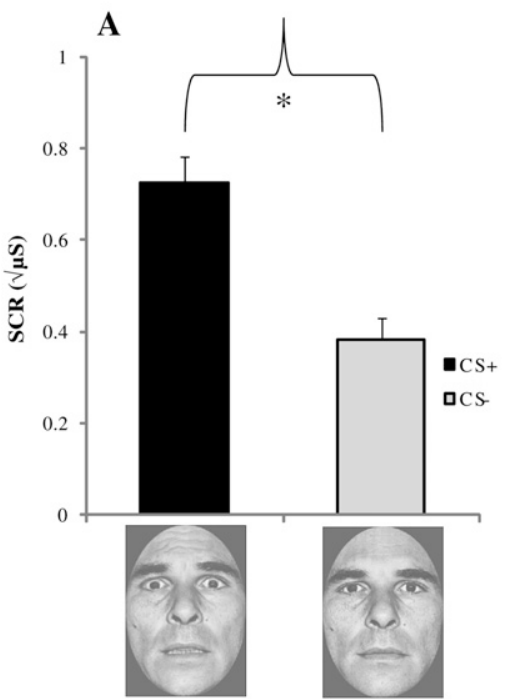

Figure 3. Experiment 1 fear conditioning results. (A) Mean skin conductance responses $(\mathrm{SCR})$ in microsiemens $(\mu S),(B)$ reaction times (RTs), and (C) subjective face ratings. Error bars reflect standard error of the mean $\left.(\mathrm{SEM}) ;{ }^{*}\right)$ denote significant differences $(P<0.05)$.

than the CS+ that were rated by participants as being neutral. The overall pattern demonstrates an asymmetrical generalization gradient around the reinforced value, consistent with the prediction of an intensity generalization gradient (Ghirlanda and Enquist 2003) but inconsistent with a perceptual feature-based generalization gradient, which would predict a bell-shaped curve around the $\mathrm{CS}+$ value. Saturated monotonic gradients occur when responses evoked by nonconditioned values of greater intensity than the CS+, along a dimension of increasing intensity, do not fall below the CS+ response level (Ghirlanda 2002; Ghirlanda and Enquist 2003). This pattern was consistent across the three blocks of the generalization test (Fig. 2B), with an overall decrease in responses, as would be expected over the course of an extended conditioning session.

\section{Pre-to-post SCR analysis}

To determine whether the stimulus generalization gradient emerged as a consequence of fear conditioning and was not simply a function of nonassociative responses to the stimulus values prior to fear conditioning, an additional analysis was conducted on the difference in SCR to all five stimulus values from preconditioning to the generalization test. ${ }^{2}$ For this analysis, participants' mean response to each stimulus value during preconditioning was subtracted from their response to the same stimulus value during generalization testing. Inspection of this difference gradient (Fig. 2A) reveals a similar shape as the generalization test gradient itself. An ANOVA of the pre-to-post SCR difference revealed a main effect

${ }^{2}$ SCRs during preconditioning may not be directly comparable to SCRs during the generalization test due to different processes that contribute to fear expression at each experimental phase. Nonetheless, it is important to show that the pattern observed during the generalization test did not exist prior to the conditioning phase. It is common in electrophysiological studies (e.g., Weinberger 2007) to compare responses with an array of stimuli along a sensory dimension prior to and following fear conditioning to make inferences about learning-induced changes in stimulus representations. for stimulus type on the magnitude of the SCR, $F_{(4,140)}=8.55, P<$ 0.001 , with a significant linear trend, $F_{(1,35)}=30.20, P<0.001$.

\section{$R T$}

Following fear conditioning, ANOVA revealed a main effect of stimulus type on RT (Fig. 2C), $F_{(4,136)}=15.77, P<0.001$, as well as both linear $(P<0.01)$ and quadratic trends $(P<0.01)$. The pattern of RTs during the generalization test was similar to that during preconditioning, such that the fastest RTs were elicited by the most intense fear face $(100 \%, \mathrm{~S} 5)$, and the slowest RTs were observed for the ambiguous stimuli (S2 and S3). There was a significant facilitation in RTs from preconditioning to the generalization test for the stimulus serving as the CS+ (S3), $t_{(34)}=$ $2.54, P<0.05$, and the $S 4, t_{(35)}=2.22, P<0.05$.

\section{Subjective face ratings}

The S1 and S2 were rated as not expressing fear on nearly all trials, whereas the S3, S4, and S5 were rated as expressing fear on the majority of trials (Fig. 2D). These results were similar to those during preconditioning, indicating that fear conditioning did not have an effect on subjective face ratings to any of the stimulus values.

\section{Retrospective CS+ identification}

Following the experimental session, participants were asked to identify which face stimulus had been paired with the US during the course of the experiment (Fig. 2E). Forty-five percent of participants correctly identified the S3 (55\% fear intensity). Interestingly, 36\% of participants falsely identified the S4 (77\% fear intensity) as being paired with the US, consistent with the SCR peak shift occurring during the generalization test. This result may indicate an illusory correlation (Tomarken et al. 1989), such that the stimulus of greater fear intensity was mistakenly thought to be paired with the US for a considerable number of participants.

To examine the relationship between memory accuracy for the identity of the CS+ and the extent of generalization, a subsequent ANOVA was conducted on SCRs during the generalization test using recognition accuracy as a between-subjects factor. There was no significant effect of recognition accuracy, $F_{(1,30)}=0.005, P>$ 0.05 , indicating that the form of the generalization gradient was similar irrespective of participants' abilities to accurately recollect the identity of the CS+.

\section{Experiment 2}

The goal of Experiment 2 was to determine the effect of discriminative fear conditioning between the CS+ and a CS- of greater fear intensity on the generalization gradient. If generalization operates without regard to fear intensity value, then fear conditioning with the most fear-intense value as the unpaired control would be expected to produce a bell-shaped gradient around the CS+, or potentially shift the gradient toward the less fear-intense S2 and S1 (Spence 1937; Hanson 1959). Conversely, if fear generalization is driven entirely by fear intensity of the nonconditioned stimulus, these discriminative fear-conditioning procedures would be expected to have little effect on the generalization gradient. In this case, the generalization gradient would be nearly identical to that observed in Experiment 1 . We predicted that discriminative fear conditioning in Experiment 2 would sharpen the form of the generalization gradient around the CS+ value, and that responses to the most fear-intense stimulus would be reduced relative to Experiment 1, reflecting learning-related stimulus control.

\section{Preconditioning}

As in Experiment 1, baseline SCRs did not significantly vary as a function of fear intensity, $F_{(4,136)}=1.08, P=0.36$ (Fig. 4 A). RTs were also similar to Experiment 1, such that a main effect of 
stimulus type on RT was found (Fig. 4C), $F_{(4,128)}=25.13, P<0.001$, as well as both linear $(P<0.01)$ and quadratic trends $(P<$ 0.01). The most fear-intense face evoked the fastest RT, whereas the ambiguous S2 and S3 evoked the slowest RT (Fig. 5B). Finally, the perceptual categorical boundary between the S2 and S3 was similar to Experiment 1 (Fig. 4D).

\section{Fear conditioning}

As shown in Figure 5, significantly larger SCRs were elicited by the CS+ (mean \pm SEM: $0.62 \pm 0.05)$ as compared with the CS $-(0.51 \pm 0.06)$, indicating that conditioning took place, $t_{(34)}=2.42, P<0.05$. Participants responded with significantly slower RTs to the CS+ $(1209.78 \mathrm{~ms} \pm$ 54.77) than the CS- $(891.34 \mathrm{~ms} \pm$ 33.17), $t_{(34)}=9.15, P<0.001$. Finally, the CS+ was rated as expressing fear on $74.7 \%$ of the trials, whereas the CS- was rated as expressing fear on $99.4 \%$ of the trials. Interestingly, the endorsement rate of fear expression for the CS+ was reduced from Experiment 1 (93\%).

\section{Generalization test}

\section{SCR analysis}

In contrast to Experiment 1, fear generalization was characterized by both linear, $F_{(1,34)}=12.57, P<0.01$, and quadratic trends, $F_{(1,4)}=13.11, P<0.01$, with the peak of the gradient closer to the reinforced S3 value. ANOVA confirmed a main effect of stimulus type on SCR magnitude, $F_{(4,136)}=9.48, P<0.01$. Posthoc $t$-tests, corrected for multiple comparisons, showed increasing responses from S1 to S2 $(P<0.05)$ and again from S2 to S3 $(P=0.027)$. The increase from S2 to S3 $(P=0.13)$ and from S3 to S4 $(P=1.8)$ were not significantly different, but there was a significant decline in responding from $\mathrm{S} 4$ to S5 $(P<0.05)$. These data show that the breadth of the intensitybased generalization gradient was reduced relative to Experiment 1 largely via truncated SCRs to the most fear-intense stimulus. However, a gradient reversal was not found. Finally, the pattern of stimulus generalization was similar across the three generalization test segments (Fig. 4B).

\section{Pre-to-post SCR analysis}

A comparison of SCRs to each stimulus value from pre- to post-fear acquisition revealed a main effect for stimulus type, $F_{(4,136)}=7.84$, $P<0.01$, with both linear, $F_{(1,34)}=16.27, P<0.01$, and quadratic, $F_{(1,34)}=12.29, P<0.01$ trends. Inspection of this difference gradient (Fig. 4A) reveals a similar shape as the generalization test gradient itself. A $2 \times 5$ repeated-measures ANOVA was also conducted to directly compare differences in pre-to-post SCRs across Experimental groups (Experiment: 1, 2; fear intensity: S1-S5). A significant quadratic effect was found, $F_{(1,69)}=4.68, P<0.05$, reflecting a sharper response gradient from Experiment 2 relative to Experiment 1.

\section{$R T$}

A main effect of stimulus type on RT was observed during the generalization test, $F_{(4,136)}=20.92, P<0.001$, that was both linear
$(P<0.001)$ and quadratic $(P<0.001)$. Following fear conditioning, RTs significantly increased for the least fear-intense stimulus, S1, $t_{(34)}=2.77, P<0.05$, as compared with preconditioning.

\section{Subjective face ratings}

As in Experiment 1, there was no discernable effect of fear conditioning on subjective face ratings (Fig. 4D).

\section{Retrospective CS+ identification}

Twenty-two out of 35 participants (62\%) correctly identified the $\mathrm{CS}+$ as being paired with the US during the Experiment. The S2 and S4 were falsely identified by $17 \%$ and $14 \%$ of participants, respectively, which is below chance (Fig. 4E).

To examine the effect of mistaken CS+ identification on stimulus generalization, a subsequent analysis was conducted using CS+ identification accuracy as a between-subjects factor. There was no significant effect of CS+ identification on the form of the stimulus generalization gradient, $F_{(1,32)}=0.585, P>0.05$, replicating Experiment 1.

\section{Discussion}

The results from the present study reveal broad generalization of the conditioned fear response to stimuli sharing similar physical 


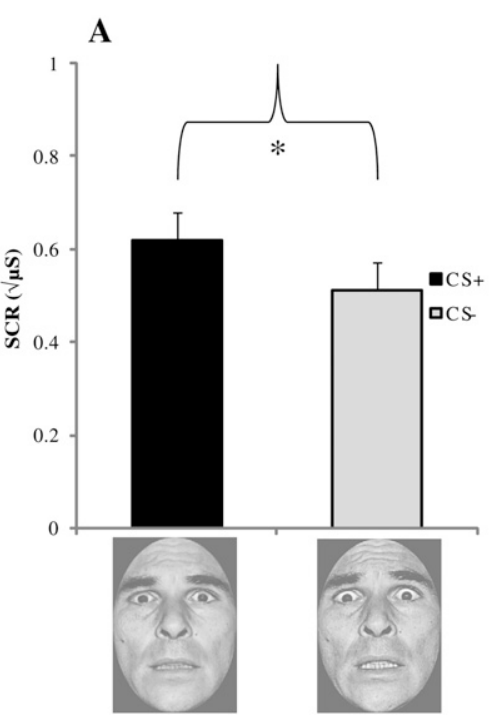

Figure 5. Experiment 2 fear conditioning results. (A) Mean skin conductance responses $(S C R)$ in microsiemens $(\mu S),(B)$ reaction times (RTs), and (C) subjective face ratings. Error bars reflect standard error of the mean $\left.(\mathrm{SEM}) ;{ }^{*}\right)$ denote significant differences $(P<0.05)$.

features to a fear-conditioned stimulus, but varying in the amount of fear intensity. Specifically, fear responses generalized from an ambiguously fearful face stimulus, reinforced with a US, to unreinforced images of the same actor displaying more or less fear expression. In line with the prediction that generalization would increase with the intensity of nonconditioned stimuli, the greatest amplitude of SCRs was recorded for stimuli that contained the highest fear intensity in Experiment 1 . This finding is complemented by the fact that a number of participants mistakenly identified a more fearful stimulus as the CS+ post-experimentally and showed increased RT to this stimulus during the generalization test. An orderly generalization gradient was also observed in Experiment 2, such that SCRs increased from stimuli containing less fear intensity than the CS+. However, the overall generalization gradient was sharper in Experiment 2 (with a quadratic trend), and the most fear-intense stimulus evoked considerably less generalization than in Experiment 1. Further, participants in Experiment 2 successfully identified the CS+ with high frequency post-experimentally, and exhibited no RT costs to the more intense expressions during the generalization test. Because Experiments 1 and 2 differed only in regard to the CS - presented during fear conditioning (Experiment 1: more fearful face, Experiment 2: less fearful face), differences in stimulus generalization between groups can be interpreted as a consequence of stimulus control gained through discrimination learning.

\section{Relationship to sensory forms of intradimensional intensity generalization}

The manner in which generalization increased with fear intensity in Experiment 1 is in line with prior nonhuman animal studies on intensity generalization (Ghirlanda 2002; Ghirlanda and Enquist 2003). When animals are trained to respond to a particular stimulus within a given sensory modality (i.e., a light or noise), there is a strong bias to respond to stimuli along the same dimension that are of greater intensity than the trained CS (i.e., increases in brightness or noise intensity) (Ghirlanda 2002). Because there was no difference in the physical intensity of the five stimuli along a sensory dimension (brightness, etc.), intensity was determined here by the degree of emotional expression, a quality defined by changes in key facial-feature configurations that constitute the display of fearful affect (Suskind et al. 2008). To our knowledge, no fear conditioning study in humans or nonhuman animals has examined intensity generalization using a CS - of higher physical intensity than the CS+. An animal study using auditory stimuli with appetitive reinforcement, though, demonstrated that linear intensity generalization gradients could be reversed when the CS - was louder than the CS+, such that less intense nonconditioned sounds elicited more generalized responses than more intense nonconditioned sounds (Huff et al. 1975). Fear generalization in the present study, however, did not show this reversed pattern (i.e., generalization was not greater to the S1 or S2 relative to the S4 in Experiment 2). This observation suggests that a dimension of fear intensity is not altogether equivalent to intensity along sensory dimensions, or that conditioned fear generalizes differently from appetitive learning. Our results further argue against a gradient interaction model for fear generalization, as inhibition to the CS-, in summation with an excitatory gradient around the $\mathrm{CS}+$, did not shift responses to values further from the CS- (Spence 1937). Thus, it is possible that fear generalization was determined to some extent by sensitization to images of fear expression.

\section{Potential role of sensitization processes in fear generalization}

Because fear-relevant stimuli may naturally evoke greater responses and be more resistant to extinction than fear-irrelevant stimuli, it is important to differentiate selective sensitization effects from associative fear learning processes in fear generalization (Lovibond et al. 1993). For instance, it has been argued that increases in general arousal sensitize fear-relevant stimuli in a nonspecific (i.e., nonassociative) manner (for review, see Öhman and Mineka 2001). It is possible that the dimension of increasing fear expression constitutes a gradient of selectively sensitized stimuli, and thus, the mere presentation of the US is sufficient to sensitize the most fearintense stimulus (i.e., pseudo-conditioning). In this case, selective sensitization may provide an account for the asymmetric gradient observed in Experiment 1. There was no evidence for a prepotent response bias along this dimension during preconditioning (i.e., the most fear-intense stimulus did not evoke larger SCRs than the least fear-intense stimulus), but fear conditioning may have increased the tonic level of arousal, which in turn may have increased sensitivity to the range of nonconditioned stimuli during the generalization test. However, the fact that stimulus generalization was markedly different between experimental groups suggests an effect of discriminative fear learning on subsequent generalization. The most striking difference is in the large decrease in responding to the most fear-intense stimulus in Experiment 2 relative to Experiment 1 . Had fear generalization operated solely as a property of selective sensitization to stimuli of high fear intensity value, without regard to prior learning, we would expect substantial generalization to the S5 in both groups of participants.

It has been argued that sensitization processes are not mutually exclusive from associative learning processes, and that sensitization may enhance the effect of associative fear learning (Rosen and Schulkin 1998). Our results may be compatible with this hypothesis. For instance, the linear gradient in Experiment 1 favors an intensity generalization account. Intensity generalization, in the context of fear expression, is likely sensitive to degrees 
of fear relevance of nonconditioned stimuli, which may be an important factor in driving fear generalization. Likewise, the gradient in Experiment 2 extended beyond the CS+ to a more fear-intense, nonconditioned stimulus (S4). In both cases, the degree of fear intensity contained within nonconditioned stimuli influenced the generalization gradient. Yet, sensitization does not fully explain why different generalization gradients emerged for Experiments 1 and 2, nor does it account for the differences in false recognition of the CS+ between experimental groups. These results suggest an influence for associative learning processes on fear generalization.

Altogether, sensitization likely has an important role in generalizing fears to nonconditioned stimuli and likely facilitates the extreme overgeneralization symptomatic of pathological anxiety (Grillon and Morgan 1999; Mineka and Zinbarg 2006). Associative learning processes, on the other hand, have a considerable role in originally acquiring conditioned fear, and it may be through associative learning processes that fear responses come under stimulus control. The full contribution of associative and nonassociative processes in fear generalization merits further investigation.

\section{Contribution of discriminative fear learning on stimulus generalization}

The difference in stimulus generalization gradients and retrospective CS+ identification in Experiments 1 and 2 suggests that fear generalization may be influenced by the representation of the CS+ formed during discriminative fear learning. An elemental model of stimulus generalization may provide an account for how the representation of the CS+ was formed over the course of fear conditioning. First, elemental models of Pavlovian conditioning have argued that the numerous elements comprising a CS can form independent associations with the US (Rescorla and Wagner 1972; Wagner 2008). Over the course of learning trials, different elements can either gain or lose associative strength, depending on which elements better predict the US. In an extension of this elemental model formulated by McLaren and Mackintosh (2002), when an organism encounters a stimulus that has not been conditioned, generalization is determined by the associative strength of the elements shared with a similar conditioned stimulus. According to this view, the elements that constitute a stimulus contain overlapping gradients of activity (Blough 1975). Through conditioning, some of these elements gain associative strength, thus increasing the excitatory gradient surrounding a particular stimulus element. Similarity is based on how many of these elements overlap between the CS+ and a nonconditioned stimulus. If elements common to both stimuli are those most associated with the US (through CS-US pairing), then stimulus generalization is likely to occur. Further, differential reinforcement between the CS+ and CS- establishes which particular elements gain associative value, while elements common only to the CS+ gain associative value, elements common to the CS - or shared between CSs lose associative value. Thus, a nonconditioned stimulus may evoke greater responding than even the CS+ (i.e., peak shift) if it is in greater possession of those elements that originally differentiated the CS+ from the CS- (McLaren and Mackintosh 2002).

In terms of the present study, discriminative fear learning established that features common to the CS+ became associated with the US, whereas features common to the CS- did not become associated with the US. For Experiment 1, in which the CS- was a relatively neutral stimulus, features that differentiated the CS+ from the CS - were those associated with fear expression, while other features related to subject identity contained the most overlap and were not predictive of the US. Consequently, fear expression predicted the US, which may account for generalization to stimuli of higher fear intensity than the CS+ following fear conditioning. On the other hand, in Experiment 2, fear expression was a poor predictor for the US, since the CS- contained more fear expression than the CS+. Consequently, features related to fear expression should have acquired very little associative strength, which may account for the decrease in SCRs to the most fearintense stimulus in this experimental group. However, while responses to the most fear-intense stimulus were reduced relative to Experiment 1, the generalization gradient was not simply the reverse of Experiment 1, as would be predicted if fear generalization were based purely on the interaction between excitatory and inhibitory elements. It is possible that conditioning an ambiguously fearful face increased the associative value of fear-relevant features in both experimental conditions, but that this propensity to generalize to fear-intense stimuli interacted with learned discrimination in Experiment 2. It may therefore be of interest in future investigations to examine fear generalization to face stimuli along other emotional (e.g., anger or happy) or neutral (e.g., identity) dimensions to further assess how the nature of the CS+ impacts fear generalization. These results suggest that fear generalization along an intradimensional gradient of fear expression is subject to particular response biases not accounted for by elemental theories or gradient interaction theories of stimulus generalization.

\section{False recognition of the fear-conditioned stimulus}

One such bias we observed was in the participants' retrospective identification of the CS+. When asked to identify which stimulus had been paired with the US during the experimental session, a large number of participants mistakenly identified a more fearful stimulus as the CS+ in Experiment 1. A related type of covariation bias has been reported in prior research (Öhman and Mineka 2001). For example, when fear-relevant and fear-irrelevant stimuli are randomly associated with an electric shock, the fear-relevant stimuli are stated as having been paired with the shock with greater frequency (Tomarken et al. 1989). A false memory for the CS+ may also reflect that participants were less attentive to the absolute features that constituted the CS+ in Experiment 1. Participants in Experiment 2 , on the other hand, correctly identified the CS+ with high frequency, suggesting that a more accurate representation for the CS+ was formed during discriminative fear conditioning. Perceptual learning between related stimuli has been shown to be enhanced by differential aversive reinforcement, leading to a greater discrimination for the CS+ (Li et al. 2008). In this case, differentiation between two stimuli that were consistently endorsed as "fear expressive" may have resulted in greater discrimination for the $55 \%$ fear exemplar CS+.

It is important to note that the representation for the CS+ did not differentially impact the gradient of SCRs within each experimental group. In other words, generalization was similar between those subjects that correctly vs. incorrectly identified the CS+, retrospectively. Thus, fear generalization is not simply determined by the ability to perceptually identify the fear-conditioned stimulus. This relationship between autonomic responses and the ability (or inability) to accurately identify a feared stimulus may be related to the role of awareness in fear conditioning (Lovibond and Shanks 2002; Knight et al. 2009). It may therefore be of interest in future studies of fear generalization to gather subjective ratings of contingency awareness and US expectancy throughout the experimental session (Dunsmoor et al. 2008).

\section{Clinical implications}

These findings have implications for certain anxiety disorders characterized by overgeneralization of fear responses to nonthreatening stimuli, such as post-traumatic stress disorder (PTSD) and specific phobias. Prior fear-conditioning studies have shown 
that individuals with PTSD fail to limit responses to the reinforced CS+ (Grillon and Morgan 1999). In another study, extinction to a cue that was perceptually similar to the CS+ did not result in generalization of inhibition to the originally conditioned stimulus (Vervliet et al. 2005). Vansteenwegen et al. (2007) showed that individuals fearful of spiders failed to generalize extinction of a feared spider exemplar to a novel context when provided with extinction training in a single context. However, a group of spideranxious individuals who received extinction under multiple contexts did generalize extinction of the feared stimulus (i.e., expressed less fear renewal than the group trained in one context) when it was presented in a novel context. Clearly, the mechanisms underlying fear generalization are complex and involve responses to features related both to the context and the cue-predicting threat. It is noteworthy that the present study used a relatively ambiguous fear stimulus as the CS+ (near the point of subjective equality between emotion categories), which was sufficient to yield broad generalization gradients and false memories for the intensity of the CS+ (Experiment 1).

Finally, the limited numbers of human fear generalization studies to date have supported the idea that fear excitation is broadly tuned, whereas fear inhibition is selective to the CS(Vervliet et al. 2004, 2005; Lissek et al. 2008). This capacity to broadly generalize fear may serve as an adaptive function to aid survival in an ever-changing, potentially harmful environment (Mineka 1992). In the present study, a reduction in SCRs to the CS - resulting from discriminative fear conditioning remained specific to the CS- exemplar. These findings indicate that generalization is conserved across a wide range of perceptually similar stimuli, whereas specialization-learning when not to respond to nonthreatening stimuli-may require additional training procedures (Huff and LaBar 2009). Thus, learning to control fear responses to nonthreatening stimuli may require extinguishing exemplars of high fear intensity that are perceptually related to a feared stimulus under multiple contexts in order to overcome a predisposed (or exacerbated) fear generalization process.

\section{Conclusion}

Empirical findings on stimulus generalization date back to the earliest research on classical conditioning (Pavlov 1927). Several decades' worth of animal research, which often incorporated instrumental conditioning procedures, further extended our knowledge on processes contributing to stimulus generalization. Combined with theoretical explanations attempting to distinguish between discrimination and generalization processes (Bush and Mosteller 1951; Shepard 1987; Pearce 1994), this area of research has been active for nearly a century (Ghirlanda and Enquist 2003). Yet, despite its theoretical relevance to understanding human fear learning and anxiety disorders, the role of generalization in the acquisition and expression of learned fear has received little attention in human conditioning research beyond the initial case of "Little Albert" (Watson and Rayner 1920). The present examination shows that fear generalization is not solely determined by the physical similarity between a conditioned and nonconditioned stimulus. Other factors involved in fear learning and fear expression help determine the extent to which humans generalize fear, even to relatively ambiguous exemplars. These factors include a bias to generalize to nonconditioned stimuli that contain relatively more fear intensity than a physically similar conditioned stimulus. Importantly, this process can come under stimulus control through discriminative fear learning procedures, at least in healthy adults, suggesting that fear generalization processes are partly associative in nature. The present findings have implications for behavioral approaches to treating some anxiety disorders. Future investigations will need to address the processes involved in the maintenance of fear generalization over time, the relative contribution of associative and nonassociative mechanisms, and the direct role of awareness on the expression of fear generalization in humans.

\section{Materials and Methods}

\section{Participants}

Seventy-one healthy adult volunteers ( 37 female; median age $=21$; age range $=18-33 \mathrm{yr}$ ) who were either Duke University students or members of the local community participated in this study and received either course credit or were paid $\$ 10$ for the hour-long experimental session. An additional 12 participants were excluded from analysis due to excessively low SCRs. Participants were randomly divided into two experimental groups that differed only in regard to the unreinforced control stimulus (CS-) used during the fear-conditioning phase of the experimental session. Experiment 1 consisted of 36 participants ( 21 female, median age $=20$ ) who received discriminative fear conditioning between an $55 \%$ fear intensity CS+ and an 11\% fear intensity CS-. Experiment 2 consisted of 35 participants (16 female, median age $=22$ ) who received fear conditioning with a $55 \%$ fear intensity $\mathrm{CS}+$ and a $100 \%$ fear intensity CS-. Participants completed a personal history form and were excluded from analysis for self-reported history of neurologic and psychiatric illness, substance abuse, and current medication use. All participants provided written informed consent approved by the Duke University Institutional Review Board.

\section{Stimulus set}

A male face morphed along a gradient from neutral-to-fearful was used as the stimulus set. This identity was taken from the Ekman pictures of facial affect (Ekman and Friesen 1976) and was morphed along a continuum from neutral-to-fearful using MorphMan 2000 software (STOIK) as described by LaBar et al. (2003). Face morphs were positioned in a full-frontal orientation and cropped to include only the face, without hair, ears, or neckline. Images were normalized for contrast and luminance and appeared on a gray rectangular background. Participants were exposed to the same face identity during all experimental phases to ensure that only fear expression and not other features related to actor identity (i.e., marking specific to an individual face such as moles or facial hair), was manipulated. Five faces along the continuum were used: $11 \%$ fear $/ 88 \%$ neutral, 33\% fear $/ 66 \%$ neutral, $55 \%$ fear/ $44 \%$ neutral, $77 \%$ fear $/ 2 \%$ neutral, and $100 \%$ fear. These stimuli are also labeled as S1, S2, S3, S4, and S5, respectively. Hence, the S3 served as the conditioned stimulus (CS+), and either the S1 (Experiment 1) or S5 (Experiment 2) served as the unpaired CSduring fear conditioning.

\section{Task design and procedure}

All stimuli were presented on a 17-inch flat panel monitor. The presentation of all stimuli, recording of subjective face ratings, reaction times, and shock delivery were managed with Presentation software (Neurobehavioral Systems).

Prior to the start of the experimental session, the intensity of the electric shock (US) delivered to the wrist was calibrated for each participant. Participants were always given at least two stimulations during workup to find a level deemed by the participant as being "highly annoying but not painful." While some participants required more than two stimulations to reach this subjective level, no more than five stimulations were ever applied. The participants' task involved rating whether or not face stimuli were expressing fear (forced choice, yes/no) and responding as quickly and accurately as possible by pressing one of two buttons. The order of button presses was counterbalanced between participants. Participants were not informed of the CS-US contingency at any time during the experiment, but were told that some of the faces may be followed by a shock. Participants were provided a chance to practice the subjective rating task with one 
presentation of each of the five face stimuli prior to the start of the experiment.

The experiment was separated into three phases that occurred in the same order for each participant: preconditioning (30 trials), fear conditioning (20 trials), and generalization testing (45 trials) (see Fig. 1). Stimuli were presented for $4 \mathrm{~s}$ in all phases, followed by a 500-ms blank background. The blank background was then followed by a variable duration intertrial interval (ITI), which consisted of a fixation cross. The order of all face stimuli was counterbalanced between participants and pseudo-randomized such that no more than two of the same stimulus values occurred in a row. A 5-6-min break followed the first two phases of the experiment, wherein participants passively viewed a silent video of a train traveling through British Columbia (Highball Productions). Following the generalization test, participants were shown the five face stimuli used during the study and asked to identify which face had been paired with the US during the experiment. To ensure that participants would not simply point out an intermediate value along the continuum, the collection of five stimulus values was shown to the subject in a randomized, nonlinear order (i.e., not in sequence from neutral-to-fearful).

The specific parameters for each phase are described below.

\section{Preconditioning}

Preconditioning contained six trials of each face stimulus (30 total) separated by an ITI that varied between 5,6 , and $7 \mathrm{~s}$. The US was never presented during preconditioning, but participants were still instructed that some of the faces may be followed by the US. This phase was identical for Experiments 1 and 2. This phase allowed for a baseline measure of SCRs to the five stimulus values prior to fear conditioning.

\section{Fear conditioning}

Fear conditioning involved 10 trials of the CS+ and 10 trials of the CS- separated by an ITI that varied between 8,9 , and $10 \mathrm{~s}$. The S3 (55\% fear intensity) served as the CS+ in both Experiment 1 and Experiment 2 during fear conditioning and coterminated with the US on six out of 10 trials. Importantly, in Experiment 1 the unpaired CS - was the S1 (11\% fear intensity), while in Experiment 2 the CS- was the S5 (100\% fear intensity). The CS- was never paired with the US. Only the CS+ and CS - appeared during fear conditioning.

\section{Generalization testing}

Generalization testing consisted of nine trials of each stimulus (45 total) separated by an ITI that varied between 6,7 , and $8 \mathrm{~s}$. The generalization test was broken into three segments, each containing three presentations of each of the five stimuli. Only a brief break ( $<1 \mathrm{~min})$ was given between generalization test segments. The CS+ (S3) was intermittently paired with the US on 33\% of generalization test trials ("steady-state" generalization test) (Honig and Urcuioli 1981). These steady-state ("booster" trials) reinforcement procedures are intended to extend the length of time over which responding can be measured and offset the effects of extinction and habituation (Mednick and Freedman 1960; Honig and Urcuioli 1981; Smith et al. 2006; Lim and Pessoa 2008). One unpaired US was also delivered at the start of the second and third segment of the generalization test prior to any stimulus presentation to maintain general arousal. The generalization test phase was identical for Experiments 1 and 2.

\section{SCR and RT}

SCRs were recorded with the MP-100 system (BIOPAC systems) and sampled at $200 \mathrm{~Hz}$. A conductive saline-based gel was used with $\mathrm{Ag} / \mathrm{AgCl}$ electrodes that were placed on the middle phalanx of the second and third digits of the left hand. SCR analysis was carried out on AcqKnowledge software (BIOPAC systems). SCRs were considered related to stimulus presentation if the trough-topeak response (1) occurred 1-4 s following stimulus onset, (2) lasted between 0.5 and $5.0 \mathrm{~s}$, and (3) was greater than 0.02 microsiemens $(\mu \mathrm{S})$. If these criteria were not met, the SCR was scored as zero. SCRs were hand analyzed by a trained scorer to ensure that responses were related to stimulus presentation and were not due to fluctuations occurring prior to stimulus onset, movement artifacts, or other noise in the SCR waveform as previously described (LaBar et al. 2004). Twelve participants were characterized as "nonresponders" based on a lack of measurable SCR and were therefore removed from data analysis (LaBar et al. 2004). SCRs were square-root transformed and averaged for each participant.

Mean RTs occurring prior to $100 \mathrm{~ms}$ or after $2000 \mathrm{~ms}$ from stimulus onset were not included for analysis. RTs not meeting these criteria constituted $<1 \%$ of total trials. SCR and RT data were analyzed by ANOVA and polynomial trend analyses, with an $\alpha$ value of 0.05

\section{Acknowledgments}

We thank Matthew Fecteau for assistance with the psychophysiological monitoring equipment. This study was supported by National Science Foundation grant 0745919 and National Institutes of Health grant 2 P01 NS041328.

\section{References}

Blough DS. 1975. Steady state data and quantitative model of operant generalization and discrimination. J Exp Psychol Anim Behav Process 1: 3-21.

Bush RR, Mosteller F. 1951. A model for stimulus generalization and discrimination. Psychol Rev 58: 413-423.

Dimberg U, Öhman A. 1996. Behold the wrath: Psychophysiological response to facial stimuli. Motiv Emot 20: 149-182.

Dunsmoor JE, Bandettini PA, Knight DC. 2008. Neural correlates of unconditioned response diminution during Pavlovian conditioning. Neuroimage 40: 811-817.

Ekman P, Friesen WV. 1976. Measuring facial movement. J Nonverb Behav 1: $56-75$.

Ghirlanda S. 2002. Intensity generalization: Physiology and modelling of a neglected topic. J Theor Biol 214: 389-404.

Ghirlanda S, Enquist M. 2003. A century of generalization. Anim Behav 66: $15-36$.

Graham R, Devinsky O, LaBar KS. 2007. Quantifying deficits in the perception of fear and anger in morphed facial expressions after bilateral amygdala damage. Neuropsychologia 45: 42-54.

Grillon C, Morgan CA. 1999. Fear-potentiated startle conditioning to explicit and contextual cues in Gulf war veterans with posttraumatic stress disorder. J Abnorm Psychol 108: 134-142.

Guttman N, Kalish HI. 1956. Discriminability and stimulus generalization. J Exp Psychol 51: 79-88.

Guttman N, Kalish HI. 1958. Experiments in discrimination. Sci Am 198: 77-82.

Hanson HM. 1959. Effects of discrimination training on stimulus generalization. J Exp Psychol 58: 321-334.

Honig WK, Urcuioli PJ. 1981. The legacy of Guttman and Kalish (1956) Twenty-five years of research on stimulus generalization. J Exp Anal Behav 36: 405-445.

Hovland CI. 1937. The generalization of conditioned responses. IV. The effect of varying amounts of reinforcement upon the degree of generalization of conditioned responses. J Exp Psychol 21: 261-276.

Huff NC, LaBar KS. 2009. Generalization and specialization of conditioned learning. In Generalization of knowledge: Multidisciplinary perspectives (eds. MT Banich and D Caccamise). Psychology Press, New York (in press).

Huff CI, Sherman JE, Cohn M. 1975. Some effects of response-independent reinforcement on auditory generalization gradients. J Exp Anal Behav 23: $81-86$.

Hull CL. 1943. Principles of behavior. Appleton-Century-Crofts, New York.

Jenkins HM, Harrison RH. 1962. Generalization gradients of inhibition following auditory discrimination learning. J Exp Anal Behav 5: 435 441.

Knight DC, Waters NJ, Bandettini PA. 2009. Neural substrates of explicit and implicit fear memory. Neuroimage 45: 208-214.

LaBar KS, Crupain MJ, Voyvodic JT, McCarthy G. 2003. Dynamic perception of facial affect and identity in the human brain. Cereb Cortex 13: $1023-1033$.

LaBar KS, Cook CA, Torpey DC, Welsh-Bohmer KA. 2004. Impact of healthy aging on awareness and fear conditioning. Behav Neurosci 118: 905-915.

Lanzetta JT, Orr SP. 1980. Influence of facial expressions on the classicalconditioning of fear. J Pers Soc Psychol 39: 1081-1087.

Lashley KS, Wade M. 1946. The Pavlovian theory of generalization. Psychol Rev 53: 72-87. 
Li W, Howard JD, Parrish TB, Gottfried JA. 2008. Aversive learning enhances perceptual and cortical discrimination of indiscriminable odor cues. Science 319: 1842-1845.

Lim SL, Pessoa L. 2008. Affective learning increases sensitivity to graded emotional faces. Emotion 8: 96-103.

Lissek S, Biggs AL, Rabin SJ, Cornwell BR, Alvarez RP, Pine DS, Grillon C. 2008. Generalization of conditioned fear-potentiated startle in humans: Experimental validation and clinical relevance. Behav Res Ther 46: 678687.

Lovibond PF, Shanks DR. 2002. The role of awareness in Pavlovian conditioning: Empirical evidence and theoretical implications. J Exp Psychol Anim Behav Process 28: 3-26.

Lovibond PF, Siddle DAT, Bond NW. 1993. Resistance to extinction of fearrelevant stimuli-preparedness or selective sensitization. J Exp Psychol Gen 122: 449-461.

McLaren IPL, Mackintosh NJ. 2002. Associative learning and elemental representation: II. Generalization and discrimination. Anim Learn Behav 30: $177-200$.

Mednick SA, Freedman JL. 1960. Stimulus-generalization. Psychol Bull 57: 169-200.

Mineka S. 1992. Evolutionary memories, emotional processing, and the emotional disorders. In The psychology of learning and motivation: Advances in research and theory (ed. DL Medin),Vol. 28, pp. 161-206. Academic Press, New York.

Mineka S, Zinbarg R. 2006. A contemporary learning theory perspective on the etiology of anxiety disorders-it's not what you thought it was. Am Psychol 61: 10-26.

Öhman A, Mineka S. 2001. Fears, phobias, and preparedness: Toward an evolved module of fear and fear learning. Psychol Rev 108: 483-522.

Öhman A, Soares JJF. 1998. Emotional conditioning to masked stimuli: Expectancies for aversive outcomes following nonrecognized fearrelevant stimuli. J Exp Psychol Gen 127: 69-82.

Öhman A, Erixon G, Löfberg I. 1975. Phobias and preparedness: Phobic versus neutral pictures as conditioned stimuli for human autonomic responses. J Abnorm Psychol 84: 41-45.

Öhman A, Esteves H, Soares JJF. 1995. Preparedness and preattentive associative learning-electrodermal conditioning to masked stimuli. $J$ Psychophysiol 9: 99-108.

Öhman A, Flykt A, Esteves F. 2001. Emotion drives attention: Detecting the snake in the grass. J Exp Psychol Gen 130: 466-478.

Pavlov IP. 1927. Conditioned reflexes. Oxford University Press, Oxford, UK.

Pearce JM. 1987. A model for stimulus generalization conditioning. Psychol Rev 94: 61-73.

Pearce JM. 1994. Similarity and discrimination: A selective review and a connectionist model. Psychol Rev 101: 587-607.
Rescorla RA, Wagner AR. 1972. A theory of Pavlovian conditioning: Variations in the effectiveness of reinforcement and nonreinforcement. In Classical conditioning: II. Current research and theory (eds. AH Black and WF Prokasy), pp. 64-99. Appleton-Century-Crofts, New York.

Rosen JB, Schulkin J. 1998. From normal fear to pathological anxiety. Psychol Rev 105: 325-350.

Sabatinelli D, Bradley MM, Fitzsimmons JR, Lang PJ. 2005. Parallel amygdala and inferotemporal activation reflect emotional intensity and fear relevance. Neuroimage 24: 1265-1270.

Shepard RN. 1987. Toward a universal law of generalization psychological science. Science 237: 1317-1323.

Smith SD, Most SB, Newsome LA, Zald DH. 2006. An emotion-induced attentional blink elicited by aversively conditioned stimuli. Emotion 6: 523-527.

Spence KW. 1937. The differential response of animals to stimuli differing within a single dimension. Psychol Rev 44: 430-444.

Suskind JM, Lee DH, Cusi A, Feiman R, Grabski W, Anderson AK. 2008. Expressing fear enhances sensory acquisition. Nat Neurosci 11: 843-850.

Tomarken AJ, Mineka S, Cook M. 1989. Fear-relevant selective associations and covariation bias. J Abnorm Psychol 98: 381-394.

Vansteenwegen D, Vervliet B, Iberico C, Baeyens F, Van den Bergh O, Hermans D. 2007. The repeated confrontation with videotapes of spiders in multiple contexts attenuates renewal of fear in spider-anxious students. Beh Res Ther 45: 1169-1179.

Vervliet B, Vansteenwegen D, Eelen P. 2004. Generalization of extinguished skin conductance responding in human fear conditioning. Learn Mem 11: $555-558$.

Vervliet B, Vansteenwegen D, Baeyens F, Hermans D, Eelen P. 2005. Return of fear in a human differential conditioning paradigm caused by a stimulus change after extinction. Behav Res Ther 43: 357-371.

Vervliet B, Vansteenwegen D, Eelen P. 2006. Generalization gradients for acquisition and extinction in human contingency learning. Exp Psychol 53: $132-142$.

Wagner AR. 2008. Evolution of an elemental theory of Pavlovian conditioning. Learn Behav 26: 253-265.

Watson JB, Rayner R. 1920. Conditioned emotional reactions. J Exp Psychol 3: $1-14$.

Weinberger NM. 2007. Associative representational plasticity in the auditory cortex: A synthesis of two disciplines. Learn Mem 14: 1-16.

Whalen PJ, Rauch SL, Etcoff NL, McInerney SC, Lee MB, Jenike MA. 1998. Masked presentations of emotional facial expressions modulate amygdala activity without explicit knowledge. J Neurosci 18: 411-418.

Received March 24, 2009; accepted in revised form April 30, 2009. 


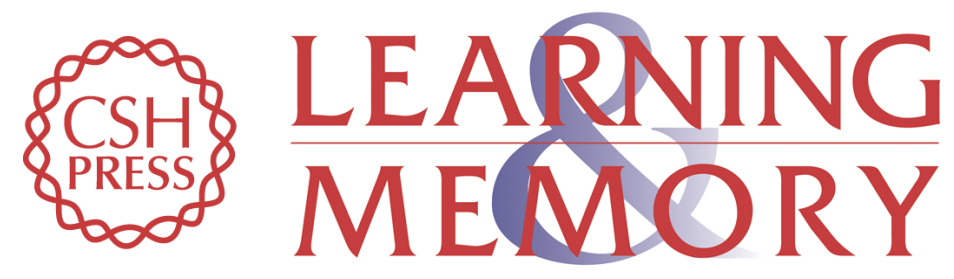

\section{Generalization of conditioned fear along a dimension of increasing fear intensity}

Joseph E. Dunsmoor, Stephen R. Mitroff and Kevin S. LaBar

Learn. Mem. 2009, 16:

Access the most recent version at doi:10.1101//m.1431609

References This article cites 52 articles, 5 of which can be accessed free at:

http://learnmem.cshlp.org/content/16/7/460.full.html\#ref-list-1

License

Email Alerting Receive free email alerts when new articles cite this article - sign up in the box at the Service top right corner of the article or click here. 